\title{
SISTEMA DE UTILIZACIÓN DE MEDICAMENTOS EN PACIENTES DE ALTO RIESGO FARMACOLÓGICO: RETO DE CALIDAD
}

\author{
Sandra Beatriz Escobar Espinosa MD*, Rocío Moreno de Santacruz**, Nathaly Rivera Romero***
}

\section{Resumen}

El sistema de utilización de medicamentos (SUM) está compuesto por múltiples procesos donde interactúan enfermeras, médicos y personal de farmacia, siendo las primeras quienes tienen más responsabilidades. Este estudio buscó establecer la prevalencia y posibles causas de los errores en los pacientes de alto riesgo farmacológico en un hospital de alta complejidad. Método: estudio de corte transversal con seguimiento durante tres meses al SUM, con un total de 318 adultos; se revisó el cumplimiento en la formulación, entrega, preparación y administración de los medicamentos. Los datos se obtuvieron mediante observación en campo y revisión de historias clínicas. Resultados: la prevalencia global de error por cada 100 dosis/ pacientes en todo el SUM fue de $5 \%$ o más en $95 \%$ de los casos. Del total de errores reportados, $\mathbf{1 . 8 9 \%}$ llegó a causar daño al paciente. Los turnos de la mañana y el fin de semana, la experiencia menor de seis meses del personal de enfermería y la estancia hospitalaria mayor de diez días fueron situaciones con mayor prevalencia. Conclusión: la prevalencia superior de errores en pacientes de alto riesgo farmacológico sugiere una mayor dificultad en el manejo de medicamentos en estos casos. Las estrategias de mejoramiento deben estar alineadas con el contexto institucional y ser específicas para este grupo de pacientes.

Palabras clave: sistemas, procesos, errores en la administración de medicamentos, alto riesgo medicamentoso. Abreviaturas: SUM, sistema de utilización de medicamentos.

\section{MEDICATION USE SYSTEM FOR PATIENTS IN THE HIGH PHARMACOLOGICAL RISK GROUP: QUALITY CHALLENGE}

\begin{abstract}
The medication use system (MUS) comprises multiple processes where nurses, doctors and pharmacists interact. Nurse practitioners have the greatest responsibility of the three. The aim of this study was to establish the prevalence and possible causes for medication errors in patients with high pharmacological risk in a high complexity hospital. Method: this was a three-month MUS follow-up cross-sectional study, including 318 adult participants; reviewing medication prescription compliance, delivery, preparation and administration. Data was obtained through field observation and a review of clinical records. Results: overall error prevalence per 100 doses/patients in the entire MUS was $5 \%$ or more, in $95 \%$ of the cases. Of the total amount of reported errors, $1.89 \%$ caused patient harm. Situations with the greatest prevalence were: morning and week end shifts, nurses with less than six month of experience and length of hospital
\end{abstract}

Fecha recibido: mayo 16 de 2013 - Fecha aceptado: agosto 23 de 2013

* Jefe de Epidemiología, Hospital de San José. Bogotá DC, Colombia.

** Enfermera especialista en gerencia de la salud. Profesora Titular, Fundación

Universitaria de Ciencias de la Salud, Bogotá DC, Colombia.
*** Enfermera, Servicio de Psiquiatría, Hospital de San José. Instructor Asistente. Joven investigador Colciencias. Fundación Universitaria de Ciencias de la Salud, Bogotá DC, Colombia. 
stay greater than ten days. Conclusion: the highest prevalence of errors in patients with high pharmacological risk suggests a greater difficulty in medication management. Improvement strategies need to be aligned with institutional context and be specific for this group of patients.

Key words: systems, processes, medication administration errors, higher risk of adverse drug reactions

\section{Introducción}

Los errores son comunes en los procesos de obtención, formulación, entrega, administración y control de medicamentos. El impacto es relevante, ya que los errores producen daños a un estimado de un millón y medio de personas y mueren varios miles por año en los Estados Unidos. ${ }^{1}$

En algunos países hasta el $67 \%$ de la medicación recetada en las historias clínicas tiene uno o más errores ${ }^{2}$, esto podría ser mayor en aquellos con alto riesgo farmacológico, ya que tienen características especiales: recibir más de cinco medicamentos, antibioticoterapia de amplio espectro, medicamentos de ventana terapéutica estrecha y/o presentar antecedentes de alergia a los medicamentos. Los problemas reportados con la terapia farmacológica se pueden evitar tomando medidas preventivas, entre las cuales se destaca la educación del personal de enfermería involucrado en la administración de medicamentos. ${ }^{3}$

En la normatividad legal colombiana se encuentra el decreto 1011/06 por el cual se establece el Sistema Obligatorio de Garantía de la Calidad en Salud ${ }^{4}$, donde los componentes de habilitación y acreditación centran su atención en la seguridad del paciente, basada en aspectos como la competencia del personal sanitario, la definición o adopción de guías y protocolos y el uso racional de medicamentos, entre otros. La garantía de calidad ha probado ser un medio para entregar servicios de salud de alta calidad eficaces y eficientes en función de los costos. ${ }^{5}$

La gestión de la calidad considera que todo trabajo implica procesos y sistemas. ${ }^{5}$ Un proceso puede definirse como un grupo de causas y condiciones que repetidas en conjunto se transforman en entradas y resultados ${ }^{5,6}$
Un sistema es la suma total de todos los elementos (incluyendo procesos) que interactúan para una meta común. Con el mejoramiento de los procesos se busca la reducción de la variabilidad en la práctica ${ }^{7}$ logrando así la estandarización de la misma, buscando procesos estables y resultados predecibles.

El sistema de utilización de medicamentos (SUM) está compuesto por múltiples procesos donde interactúan enfermeras, médicos y personal de farmacia, siendo enfermería la que más responsabilidades tiene. En este estudio se buscó establecer la prevalencia de los errores para los pacientes de alto riesgo farmacológico, en un hospital de alta complejidad, explorando las posibles causas.

\section{Método}

Estudio de corte transversal en el cual se realizó un seguimiento de tres meses al SUM, se obtuvieron un total de 318 pacientes adultos que cumplieron con los criterios de inclusión, en quienes se revisó el desempeño en el SUM por parte de enfermeras, médicos y personal de farmacia. Los datos se obtuvieron mediante la observación en campo y revisión de historias clínicas.

\section{Resultados}

Los pacientes en quienes se evaluó el sistema estuvo comprendido por adultos entre 18 y 95 años de edad, con una mediana de 55 años, de especialidades médicas y quirúrgicas, siendo cirugía general la especialidad tratante más frecuente con $28.43 \%$ seguida de medicina interna con $20.44 \%$ y con un amplio espectro de patologías (177 diagnósticos diferenciales). El 70\% de los pacientes tenía una o mas comorbilidades, el 42.14\% presentó una estancia hospitalaria de diez días 
o más y en promedio tenían formulados diez medicamentos al día.

Dentro de los errores en campo se encontró que en el 95\% de los casos presentaron dos o más errores, siendo el más frecuente la no realización de la ronda de seguridad por parte de enfermería (19\%) una vez aplicado el medicamento. En la revisión de historias clínicas se observa que el $85 \%$ tenía dos o más errores; el más común fue el de omisión (68.62\%) seguido del de prescripción (24.9\%). Se calculó la prevalencia global de error por cada 100 dosis/pacientes en el SUM y se evidenció que en el $95 \%$ de los pacientes fue de $5 \%$ o más. Del total de errores reportados $1.89 \%$ llego a causar daño al paciente.

En cuanto a las frecuencias de error por turno encontrados en la observación en campo, se halló que en la mañana los valores oscilaron entre 0 y 31 por paciente, y el fin de semana se presentaron entre 0 y 19 por paciente, con una mediana de 4 para ambos, siendo esta última más alta con respecto a la noche y la tarde ( 3 cada una) $p=0.004$. En cuanto a la experiencia, el personal de enfermería con menos de seis meses de práctica tuvo una mediana de errores mayor (14) respecto a las que tenían más tiempo (7.5) p=0.01. Los pacientes con estancia mayor de diez días presentaron una mediana de errores superior (17.5 días) con respecto a aquellos con cifra inferior (12 días) $\mathrm{p}=0.00$. No se evidenció que los pacientes con más comorbilidades $\mathrm{p}=0.132$, mayor edad $\mathrm{p}=0.4$ o más interacciones medicamentosas $\mathrm{p}=0.35$ presentaran una frecuencia superior de errores en el SUM.

\section{Disc usión}

Este estudio se realizó sobre pacientes con alto riesgo farmacológico. Se encontraron resultados muy variados con alta prevalencia de errores en el SUM, tanto en la observación en campo (95\% con dos o más errores), como en la revisión de historias clínicas (85\% con dos o más errores); esta última superior a la global $(67 \%$ uno o más errores). ${ }^{2}$ Lo anterior podría sugerir una mayor dificultad en el manejo de los medicamentos en este tipo de pacientes y deja en evidencia que los procesos que apoyan al SUM no se realizan de manera estándar, tienen una alta variabilidad en sus resultados y son poco predecibles. Dentro de las fallas propias del proceso, se encontró en la revisión de las historias clínicas el error por omisión como el más frecuente (68.62\%), seguido del de prescripción (24.3\%). En otros estudios las distribuciones son distintas: dosis (24\%), horario (22.9\%) medicamentos no autorizados $(13.5 \%)^{8}$, horario $(68.7 \%)$, omisión (12.6\%) y dosis equivocada (12.4\%). ${ }^{9}$ En campo los hallazgos más importantes fueron la no realización de la ronda de seguridad una vez aplicado el medicamento por parte de enfermería.

La proporción de errores que llegó a causar daño significativo al paciente fue de $1.89 \%$ y en todos los casos se pudo resolver sin dejar secuelas; sin embargo, la tasa reportada por la literatura es superior $(7.5 \%$ a $10.4 \%) .{ }^{10-13}$

Se exploraron las posibles causas que podrían estar afectando los procesos y se encontró que los turnos con más errores fueron los de las mañanas y los fines de semana. ${ }^{14}$ Los pacientes con estancia superior a 10 días y los atendidos por enfermeras con experiencia menor a seis meses presentaron mayor de cantidad de errores.

Por otro lado, la caracterización de los pacientes revela un grupo bastante heterogéneo donde no predomina una especialidad o patología en particular. La edad y la presencia de comorbilidades no mostraron diferencias significativas en cuanto a la frecuencia de errores. En otro estudio realizado en población pediátrica se encontró un hallazgo similar. ${ }^{15}$

Análogo a lo que sucede con otros procesos que le apuntan al mejoramiento continuo en la calidad, los resultados y dificultades en el SUM son variados entre las diferentes instituciones, por tanto las estrategias deben desarrollarse de manera particular y adaptada al contexto propio. ${ }^{7}$

Debe garantizarse la monitorización continua del SUM como parte del mejoramiento continuo de los procesos que lo componen ${ }^{7}$; sin embargo esto no puede convertirse en una causa de ansiedad o temor para los 
trabajadores de la salud, ni generar conductas punitivas; el objetivo es encontrar en cada deficiencia la oportunidad de cambiar y mejorar los procesos. ${ }^{15}$ Debe partirse del hecho de que el desempeño es una característica del sistema ${ }^{5}$; por tanto, deben identificarse qué partes del SUM son innecesarias, redundantes o faltantes dentro de los procesos e intentar mejorar otorgando una mayor claridad y/o simplificando los procedimientos, generando las condiciones necesarias que favorezcan su cumplimiento. En concordancia con esto último, una estrategia podría ser que las enfermeras inexpertas que tengan a cargo pacientes con alto riesgo farmacológico, deben recibir asesoría por el químico farmacéutico y las enfermeras de mayor experiencia, y a su vez disminuir las situaciones que hacen más problemáticos los turnos de las mañanas y fines de semana.

\section{Conclusiones}

Se evidencia una prevalencia superior de errores en el SUM para los pacientes de alto riesgo medicamentoso con respecto a la tasa global. Esto sugiere una mayor dificultad en el manejo de este tipo de casos y deja en evidencia que los procesos que apoyan al SUM no se realizan de manera estandarizada. Los turnos del fin de semana y las mañanas, la poca experiencia de las enfermeras y la estancia hospitalaria prolongada fueron factores relacionados con mayor prevalencia de errores. Se debe partir de la verificación de los procesos del SUM y los procedimientos que los componen para generar estrategias de mejoramiento, las cuales deben alinearse con el contexto institucional, ser específicas para este grupo de pacientes, generar las condiciones para su cumplimiento y realizar la monitorización continua del sistema.

\section{Referencias}

1. Philip Aspden, Julie A. Wolcott, J. Lyle Bootman, Linda R. Cronenwett, Preventing Medication Errors Institute of Medicine Of The National Academies. 2006, pp $26-45$.

2. Sullivan $\mathrm{C}$ et al. Medication reconciliation in the acute care setting: opportunity and challenge for nursing. Journal of nursing Care Quality, 2005. 20(2):95-98.

3. Telles, filho, Paulo Celso Prado, Cassiani de Bortoli, Silvia Helena. Administración de medicamentos: adquisición de conocimientos y habilidades requeridos por un grupo de enfermeros. Rev. Latino-Am. Enfermagem . 2004, vol.12, n.3, pp. 533-540.

4. Decreto 1011 del 2006 del Ministerio de la Proteccion Social, por el cual se establece el Sistema Obligatorio de Garantía de la Calidad en Salud.

5. Rashad Massoud, Karen Askov, Jolee Reinke, Lynne Miller Franco, Thada Bornstein, Elisa Knebel, y Catherine MacAulay. QA Monograph A Modern Paradigm for Improving Healthcare Quality. Quality Assurance Project (QAP). 2008.

6. Thomas w. Nolan, Lloyd P. Provost. Undesrtanding Variation. Quality Progress, ASQC, Milwaukee, May, 1990, pp. 70-78.

7. Paul E. Plsek, MS. Quality Improvement Methods in Clinical Medicine. Pediatrics Vol. 103, Jan, 1999, pp. 203-213.

8. Thalyta Cardoso, Alux Teixeira, Silvia Helena de Bortoli Casani. Root Analysis: Evaluation of medication Errors at a Untiversity Hospital. Rev Esc Enfermeria USP 2010, 44(1), 136-46.

9. Donald M. Berwick, M.D., M.P.P. Continuous Improvement as an Ideal in Health Care N Engl J Med 1989; 320:53-56 January 5, 1989.

10. María Cristina Baracaldo. Detección de errores en medicación en la administración de medicamentos. Universidad Nacional de Colombia. Revista Colombiana de Ciencias Químico farmacéuticas. Vol 29: 26 - 31. Febrero de 2000.

11. Ak Jha, N Prasopa-Plaizer, 1 Larizgotia, D W Bates. On Behalf of the Research Priority Setting Working Group of the WHO World Alliance for Patient Safety. Patient safety research: an overviem of the global evidence. Qual Saf Health Care 2010; 19:42-47.

12. Baker GR, Norton PG, Flintoft V, et al, The Canadian Adverse Eventes Study, the incidence of adverse events hospital patients in Canada. CMAJ 2004;170: 1678-86.

13. Miller GC, Britth HC, Valenti L. Adverse drug events in general practice patiens in Australia. Med J Aust 2006;184:321-4.

14. Bates DW, Cullen, Laird N, et al. Incidence of adverse drug events and potential adverse drug events. Implications for prevention. ADE prevention Study Group. JAMA $1995 ; 274: 29-34$.

15. Monica Vila`-de-Muga, MD, * Laura Colom-Ferrer, MD,P Mariona GonzalezHerrero, MD,P and Carles Luaces-Cubells, MD*. Factors Associated With Medication Errors in the Pediatric Emergency Department. Pediatric Emergency Care \& Volume 27, Number 4, April 2011. 